\title{
Developments in International Masterclasses
}

\section{Kenneth Cecire}

QuarkNet Center, University of Notre Dame

Notre Dame, Indiana

E-mail: kcecireand.edu

\author{
Uta Bilow ${ }^{1}$ \\ Institute for Nuclear and Particle Physics, Technische Universität Dresden \\ Dresden, Germany \\ E-mail: uta.bilowltu-dresden.de
}

International Masterclasses (IMC) have grown in a number of ways. World Wide Data Day (W2D2) and special masterclasses for International Day of Women and Girls in Science (IDWGS) are innovations that began two years ago and have taken root. W2D2 establishes new ways for high school students and teachers to engage in masterclass activities from their own classrooms. For IDWGS, a new pathway has been opened for high school girls to be encouraged in physics. New masterclass measurements beyond those for the LHC have been developed and tested, notably the MINERvA neutrino masterclass, which is the first IMC offering in neutrino physics and the first based on a Fermilab experiment. In the MINERvA measurement, students are able to study interactions of a neutrino beam with carbon nuclei, using conservation of momentum to draw conclusions. Other masterclass measurements related to Belle II and medical imaging are also in the testing stage. More neutrino masterclasses are in development as well, especially for MicroBooNE. A longer-term goal is the creation of a DUNE masterclass measurement as that facility reaches the data-taking stage.

European Physical Society Conference on High Energy Physics - EPS-HEP2019 -

10-17 July 2019

Ghent, Belgium

\section{${ }^{1}$ Speaker}

(C) Copyright owned by the author(s) under the terms of the Creative Commons 


\section{Introduction to International Masterclasses}

\subsection{The Masterclass Concept}

International Masterclasses enable high school students to work with physicist mentors becoming "particle physicists for a day." Students learn more about particle physics from a mentor before making a measurement based on authentic data from a contemporary experiment. At the end of the day, masterclass participants attend a videoconference with students from other locations, often in other countries, who have made the same measurement. Physicists at CERN or Fermilab moderate the videoconferences. International Masterclasses are held in and near March each year. The International Masterclass day is structured around an introductory presentation, a tour of laboratory facilities, analysis of authentic HEP data, and the videoconference.

\subsection{Reach of International Masterclasses}

International Masterclasses began in 2005 in Europe as a project of the European Particle Physics Outreach Group (EPPOG) with a small number of institutions. By 2019, now under the auspices of the International Particle Physics Outreach Group (IPPOG), successor to (EPPOG), it is worldwide in scope with over 50 countries represented. In 2019, over 10,000 high school students participated in 332 masterclasses at 239 institutions. They made measurements of authentic data from four Large Hadron Collider (LHC) experiments at CERN and one experiment each at Fermilab and KEK. Moderation of videoconferences was shared by CERN, Fermilab, TRIUMF, and KEK, with the largest number of these at CERN (69) and Fermilab (27). [1]

\section{Special Projects in International Masterclasses}

\subsection{Description}

International Masterclasses continue to expand in the number of masterclasses and in the geographical range of masterclass institutions. In addition, there are new opportunities for students and teachers to engage in masterclasses and new experimental particle physics measurements that go beyond the mainstay masterclass measurements from the LHC. The two special projects of International Masterclasses Central Coordination, World Wide Data Day and masterclasses for the International Day of Women and Girls in Science, present new opportunities.

\subsection{World Wide Data Day}

World Wide Data Day (W2D2) is a single day of videoconferences as the capstone to student and teacher measurements of ATLAS and CMS. The purpose is to have a compact masterclassstyle experience performed completely from school, without the need to visit a masterclass institution or even involve a physicist locally. Teachers help students to analyze data and look specifically for dimuon events. These events are easy to spot, even without making any cuts or oth- 
er adjustments to the event display. Students then measure the direction angles $\theta$ and $\phi$ of each muon by setting the display to the z-y and $x-y$ views of the event, respectively, and using a protractor. Students bin the results into $20^{\circ}$ intervals on a tally sheet; the class uses the tally sheets to make class histograms of $\theta$ and $\phi$ and to enter their results into a Google spreadsheet which displays histograms showing the combined results worldwide. The real physics discussion takes place in the W2D2 videoconference. [2] W2D2 was piloted in December 2016; it was held again in November 2017 and 2018 and was held this year on October 16. In 2018, over 1,000 students at 66 schools in 19 countries participated.

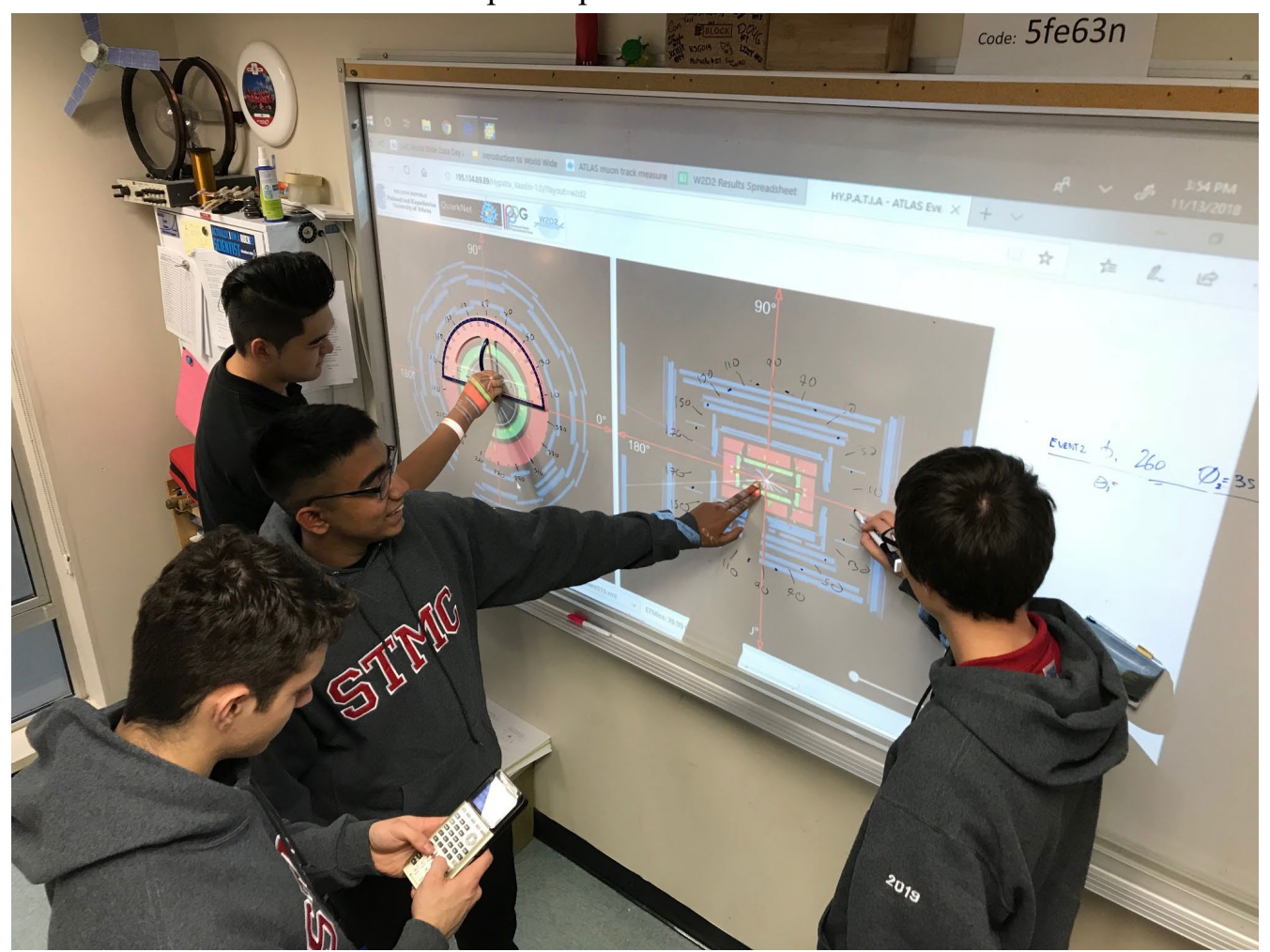

Figure 1. World Wide Data Day measurement of ATLAS data, Burnaby, Canada 2018.

\subsection{International Day of Women and Girls in Science}

Since 2016, February 11 each year has been the United Nations International Day of Women and Girls in Science (IDWGS). [3] On and around that day each year, special masterclasses for women and girls have been held, united by CERN-moderated videoconferences which focus not only on the physics, but also on issues and opportunities for women in science.

The IMC effort for IDWGS emphasizes masterclasses in a friendly physics environment for young women and women as role models for the students. Most of the participants are high school girls, and women are well represented among the masterclass tutors and organizers. The moderators at CERN are women. In 2018, there were 12 IDWGS masterclasses in five European countries plus Brazil. 


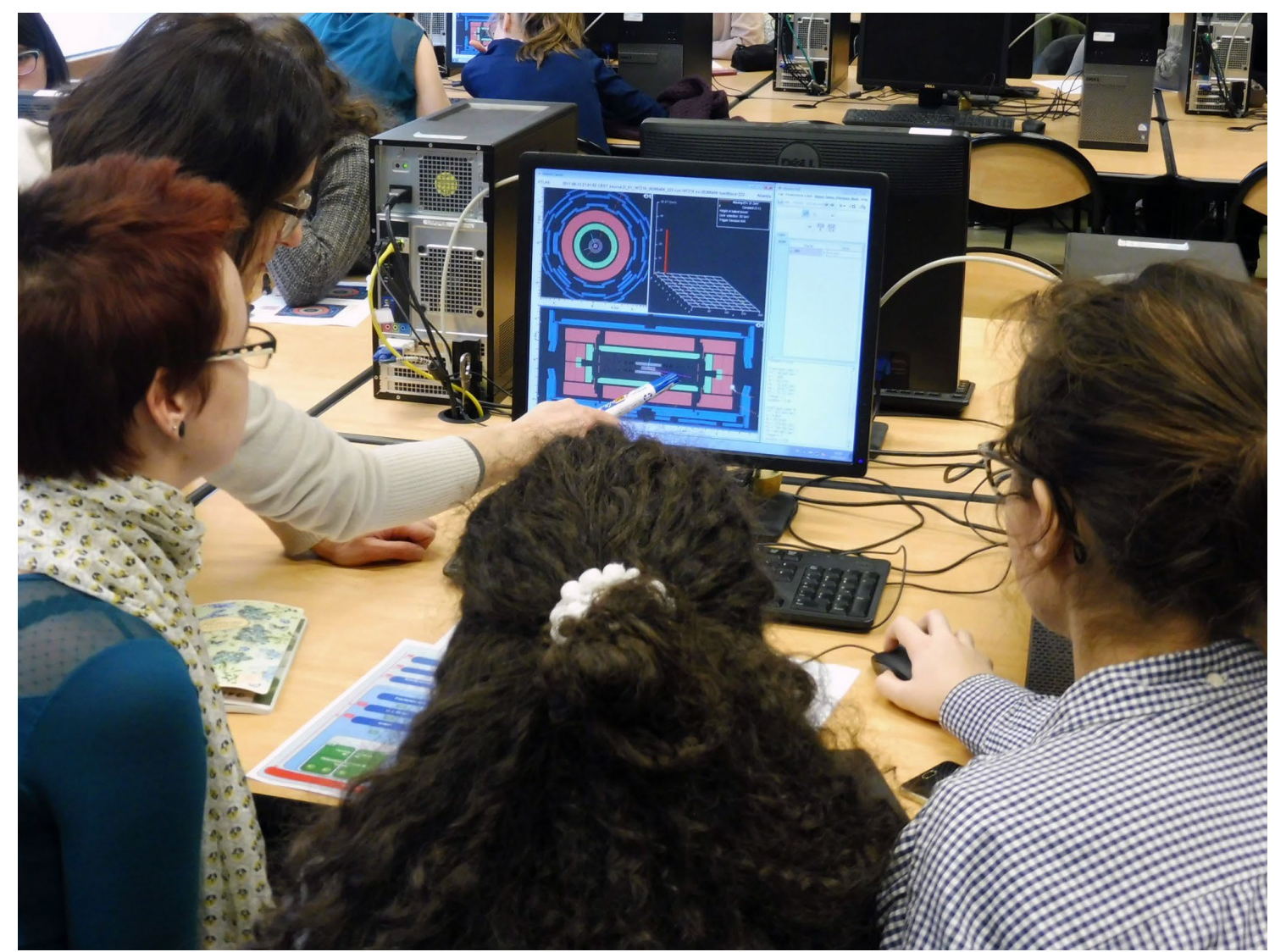

Figure 2. Students learn from a role model in a masterclass for International Day of Women and Girls in Science.

\section{New Masterclass Measurements}

\subsection{Introduction}

International Masterclasses have been focused, since 2005, on the LHC, first with Large Electron-Positron Collider masterclasses in anticipation of the LHC and then, starting in 2010, with LHC data from ALICE, ATLAS, CMS, and LHCb. Since then, some non-LHC, nonCERN groups began to develop masterclasses, notably IceCube and Pierre Auger. In 2018, development of "other-brand" measurements for International Masterclasses began in earnest. Belle II (KEK) physicists began work on the Belle II masterclass. GSI Darmstadt and the Heidelberg Ion Therapy Center began to pioneer a particle therapy masterclass. QuarkNet and Fermilab collaborated to develop and roll out accelerator-based neutrino masterclasses with the ultimate goal, still years off, of producing a masterclass for the Deep Underground Neutrino Experiment (DUNE).

\subsection{Upgrade of the CMS Masterclass}

In the past several years, the CMS masterclass used in International Masterclasses has employed the "WZH" measurement, in which students look for leptonic decays to make ratios of $\mathrm{W}^{+}$to $\mathrm{W}^{-}$candidates as well as of muons to electrons. They also make a mass plot which shows apparent $\mathrm{Z}, \mathrm{J} / \Psi$, and $\mathrm{Y}$ peaks. Students can also add a very limited number of Higgs candidates. 
In 2018, nearly 300 four-lepton events became available, showing apparent peaks for $\mathrm{Z} \gamma$ and ZZ events as well as a smaller sample close to the Higgs mass. Work is underway to incorporate the new data so that students can make meaningful two- and four-lepton mass plots from their masterclass analysis.

\subsection{Belle II}

Belle II is an upgraded, more capable version of the Belle experiment at KEK to study B physics coming from electron-positron collisions. Based on the highly successful KEK B-Lab for students, the Belle II masterclass does not focus so much on individual events as on large numbers of events. The student task, then, is to use code similar to SCRATCH in order to filter data and make a variety of plots. The Belle II masterclass was piloted in International Masterclasses 2019, improved, and is expected to be available for 2020. [5]

\subsection{Particle Therapy}

Medical physics has great potential to capture the interest of students who are more oriented toward life sciences or are more motivated by applications of science to society. Student participants examine cancer scenarios and plan treatment with photon, carbon nucleus, and proton therapies, calculating optimal doses using the open source matRad toolkit. The particle therapy masterclass will be introduced into International Masterclasses in 2020. [6]

\subsection{Low-Mass Particles in ALICE}

There are two existing masterclass measurements from the ALICE experiment in LHC. A third is under development so students can find and measure $\mathrm{J} / \Psi$ and similar low-mass mesons in heavy ion collisions in ALICE.

\subsection{Neutrino Masterclasses}

As Fermilab and other institutions have begun to push the intensity frontier with accelerator-based neutrino experiments, physicists and educators became more interested in masterclasses to bring this physics to teachers and students. To this end, collaborators at the University of Rochester began to develop a measurement about five years ago to enable students to apply momentum studies to interactions of neutrinos with carbon nuclei in the Fermilab MINERvA experiment. [7] In 2018 and 2019, QuarkNet, Rochester physicists, and Fermilab developed this further into a full masterclass, which debuted and was tested in International Masterclasses 2019. Participants examine events in the MINERvA detector, first separating signal from background and then using conservation of momentum and the uncertainty principle to test models of the interaction. The more successful model enables students to find the neutrino beam energy, Fermi motion of neutrons in the nucleus, and an approximation of the carbon nuclear radius. Student success at the measurement and interest were both high. [8]

At the same time, Fermilab MicroBooNE physicists have been collaborating with QuarkNet to develop a detector commissioning masterclass. The liquid argon time-projection chamber (TPC) technology in MicroBooNE is sophisticated and sensitive; students can, for example, examine cosmic ray muon tracks in the detector to determine the purity of the argon and the over- 
all response of the detector. The MicroBooNE masterclass will be tested in International Masterclasses 2020.

MINERvA tests models of neutrino interactions that physicists need to understand very well to make best use of DUNE when it comes online; MicroBooNE uses and enables student to test the key liquid argon TPC technology on which DUNE will rely.

This activity has spurred interest from other neutrino experiments so that several more are expected to come online in the coming years. DUNE physicists are already considering a simulated supernova measurement based on the projected capabilities of the detector.

\section{Conclusion}

\subsection{Bright, Interesting Future}

CERN and Fermilab are currently the two main centers for moderating masterclass videoconferences. In addition, TRIUMF runs a single videoconference each year for mostly local British Columbia masterclass institutes. In 2019, Fermilab added to its portfolio with MINERvA masterclasses, and KEK moderated a single videoconference for the Belle II masterclass, which is he first from that laboratory. As the new masterclasses increase and expand, KEK and Fermilab will increase their activity while new videoconference centers will come on stream.

LHC masterclasses will continue to develop, as seen with the revision of the CMS masterclass and a planned new $\mathrm{J} / \Psi$ measurement for ALICE.

The new opportunities seen with W2D2 and IDWGS continue to be strong and new opportunities continue to open.

\section{References}

[1] K. Cecire, Particle Physics Masterclasses, Proceedings of the DPF-2011 Conference, Providence, RI, August 8-13, 2011.

[2] World Wide Data Day website, https://quarknet.org/content/world-wide-data-day.

[3] United Nations General Assembly, Resolution adopted by the General Assembly on 22 December 2015 , https://www.un.org/en/ga/search/view_doc.asp?symbol=A/RES/70/212.

[4] International Masterclasses website, Intl. Day of Women and Girls in Science, https://www.physicsmasterclasses.org/index.php?cat=women_in_science.

[5] R. Pestotnik, Belle II Masterclass, Masterclass Steering Group Meeting, Darmstadt, May 2019.

[6] N. Wahl, Particle Therapy MasterClass Overview and Pilot Report, Masterclass Steering Group Meeting, Darmstadt, May 2019.

[7] Neutrino Classroom website, https://neutrino-classroom.org/.

[8] Neutrino Masterclass Project Map 2019, https://quarknet.org/content/neutrino-masterclassproject-map-2019. 